\title{
The Nucleus Accumbens and Ketamine Treatment in Major Depressive Disorder
}

\author{
Chadi G Abdallah*,1,2, Andrea Jackowski ${ }^{3}$, Ramiro Salas ${ }^{4,5}$, Swapnil Gupta ${ }^{1,2}$, João R Sato ${ }^{3,6}$, Xiangling Mao ${ }^{7}$, \\ Jeremy D Coplan ${ }^{8}$, Dikoma C Shungu ${ }^{7}$ and Sanjay J Mathew ${ }^{4,9}$ \\ 'Clinical Neuroscience Division, National Center for PTSD, VA CT Healthcare System, West Haven, CT, USA; ${ }^{2}$ Department of Psychiatry, Yale \\ University School of Medicine, New Haven, CT, USA; ${ }^{3}$ LiNC, Departamento de Psiquiatria, Universidade Federal de São Paulo, São Paulo, Brazil; \\ ${ }^{4}$ Menninger Department of Psychiatry and Behavioral Sciences, Baylor College of Medicine, Houston, TX, USA; ${ }^{5}$ Research Care Line, Michael E. \\ DeBakey VA Medical Center, Houston, TX, USA; ${ }^{6}$ Center of Mathematics, Computation and Cognition. Universidade Federal do ABC, Santo Andre, \\ Brazil; ' Departments of Radiology, Psychiatry and Biophysics, Weill Medical College of Cornell University, New York, NY, USA; ${ }^{8}$ Division of \\ Neuropsychopharmacology, Department of Psychiatry, SUNY Downstate Medical Center, Brooklyn, NY, USA; ${ }^{9}$ Mental Health Care Line, Michael \\ E. DeBakey VA Medical Center, Houston, TX, USA
}

Animal models of depression repeatedly showed stress-induced nucleus accumbens (NAc) hypertrophy. Recently, ketamine was found to normalize this stress-induced NAc structural growth. Here, we investigated NAc structural abnormalities in major depressive disorder (MDD) in two cohorts. Cohort A included a cross-sectional sample of 34 MDD and 26 healthy control (HC) subjects, with high-resolution magnetic resonance imaging (MRI) to estimate NAc volumes. Proton MR spectroscopy ('H MRS) was used to divide MDD subjects into two subgroups: glutamate-based depression (GBD) and non-GBD. A separate longitudinal sample (cohort B) included I6 MDD patients who underwent $\mathrm{MRI}$ at baseline then $24 \mathrm{~h}$ following intravenous infusion of ketamine $(0.5 \mathrm{mg} / \mathrm{kg})$. In cohort $\mathrm{A}$, we found larger left $\mathrm{NAc}$ volume in MDD compared to controls (Cohen's $d=1.05)$, but no significant enlargement in the right NAc $(d=0.44)$. Follow-up analyses revealed significant subgrouping effects on the left $(d \geqslant 1.48)$ and right NAc $(d \geqslant 0.95)$ with larger bilateral NAc in non-GBD compared to GBD and HC. NAc volumes were not different between GBD and HC. In cohort B, ketamine treatment reduced left NAc, but increased left hippocampal, volumes in patients achieving remission. The cross-sectional data provided the first evidence of enlarged NAc in patients with MDD. These NAc abnormalities were limited to patients with non-GBD. The pilot longitudinal data revealed a pattern of normalization of left NAc and hippocampal volumes particularly in patients who achieved remission following ketamine treatment, an intriguing preliminary finding that awaits replication.

Neuropsychopharmacology (2017) 42, 1739-1746; doi: I0.1038/npp.2017.49; published online 29 March 2017

\section{INTRODUCTION}

Major depressive disorder (MDD) is a common mental illness that is often chronic and disabling, with more than one-third of patients treatment resistant to traditional antidepressants (Coplan et al, 2014). A major challenge for drug development in MDD is the limited understanding of the pathophysiology of the disorder and the lack of biomarkers of treatment response (Phillips et al, 2015). The nucleus accumbens (NAc) is a brain region that plays a critical role in reward circuitry and has been extensively studied in preclinical models of chronic stress and depression (Nestler, 2015). In contrast to the hippocampus, where atrophy has been tied to depression, preclinical studies

*Correspondence: Dr CG Abdallah, Clinical Neuroscience Division, National Center for PTSD, 950 Campbell Ave I5IE, West Haven, CT 065 I6, USA, Tel: 347987 07|7, Fax: 203764 5963,

Email: chadi.abdallah@yale.edu

Received 2 November 2016; revised I February 2017; accepted 4 March 2017; accepted article preview online 8 March 2017 repeatedly demonstrated hypertrophy of the NAc in models of depression (Russo and Nestler, 2013). In this study, we investigated whether these preclinical findings of enhanced neuronal remodeling in the NAc would translate into an increase in NAc volume in MDD subjects (cohort A). We then studied, in a separate sample (cohort B), the association between NAc volume and treatment with ketamine, a rapid acting antidepressant with robust effects on neuronal remodeling (Abdallah et al, 2016a).

The synaptic hypothesis of depression proposes that depression and chronic stress reduce astrocytic glutamate reuptake in the hippocampus and prefrontal cortex leading to increased extracellular glutamate and excitotoxicity, subsequently precipitating neuronal atrophy including reductions of synaptic strength, spine density, and dendritic branching and length (Abdallah et al, 2015c). Antidepressants, in particular ketamine, are believed to exert their beneficial effects by reversing the stress-induced prefrontal and hippocampal neuronal atrophy (Duman et al, 2016). Consistent with these preclinical models, reduced prefrontal 
and hippocampal volumes in MDD patients have been demonstrated in meta-analyses (Kempton et al, 2011), though these findings were not ubiquitous across individual studies. In addition, ketamine treatment of MDD patients was recently found to robustly reverse widespread depression-related prefrontal dysconnectivity, a measure believed to parallel the stress-induced neuronal deficits (Abdallah et al, 2016b). These MRI findings could reflect changes in dendritic arborization, water content shift, or other inflammation-related abnormalities.

Intriguingly, preclinical models of depression have repeatedly shown almost the exact opposite neuroplasticity effects in the NAc, demonstrating stress-induced increased synaptic strength, spine density, and dendritic branching and length (Campioni et al, 2009; Christoffel et al, 2011; Christoffel et al, 2012; Muhammad et al, 2012; Warren et al, 2014). A recent preclinical study has confirmed that chronic unpredictable stress induces an increase in dendritic length and spine density in the NAc. In addition, the study revealed that ketamine treatment reversed stress-induced neuroplasticity alterations in the NAc (Melo et al, 2015). The question is therefore raised whether these preclinical findings in the NAc would translate into tangible evidence in patients suffering from MDD. Thus, we investigated the hypothesis that MDD would be associated with larger NAc volumes and that ketamine would induce a reduction in NAc volume in depressed patients.

In cohort $\mathrm{A}$, we previously demonstrated that gammaaminobutyric acid (GABA) levels in medial prefrontal cortex (mPFC) can be used as a robust biomarker to stratify patients into two subgroups, a glutamate-based depression (GBD) and a non-GBD (Abdallah et al, 2015a). The GBD patients have abnormally low mPFC Glx and GABA, and large hippocampal volume reduction, compared to healthy control (HC). The non-GBD have normal levels of mPFC GABA and Glx, and no hippocampal volume differences compared to HC (Supplementary Information). Therefore, we also investigated whether the GBD subgroup would show the largest increase in NAc volume. However, we note that the preclinical stress-induced NAc hypertrophy is related to maladaptive dopaminergic transmission in the NAc, which raises the alternative possibility of increased NAc volume in the non-GBD group. The latter hypothesis, if confirmed, would support a working model in which MDD patients are divided into subgroups with amino acid-based (GBD) vs monoamine-based (non-GBD) gray matter abnormalities. In contrast to the glutamate-induced excitotoxicity and hippocampal atrophy (Averill et al, 2016), the stress-induced NAc hypertrophy is related to monoamine abnormalities, in particular the dopaminergic neurotransmission in the ventral tegmental area (VTA) to the NAc pathway (Chaudhury et al, 2013). Stress and depression are believed to precipitate phasic activation of the VTA-to-NAc pathway, leading to corelease of dopamine and BDNF in the NAc (Walsh et al, 2014). Subsequently, the stress-induced BDNF release results in NAc hypertrophy and in depressive-like behavior (Wook Koo et al, 2016). Consistent with the amino acid- vs monoamine-based model, the stress-induced monoamine dysregulation is also limited to a subgroup of animals. Among rodents exposed to stress, there is a subgroup of resilient animals that do not develop NAc hypertrophy,
BDNF increases, or depressive-like behavior (Krishnan et al, 2007).

To test these hypotheses, we conducted a set of analyses in two separate cohorts. In cohort A, we compared NAc volumes in patients with MDD compared to HC. In addition, follow-up analyses compared the NAc volume between the GBD, non-GBD, and HC groups. Exploratory analyses in the MDD group examined the relationship between NAc volumes and clinical severity. In cohort $\mathrm{B}$, we investigated the effects of ketamine on NAc volume in MDD patients, followed by post-hoc analyses exploring whether the effects of ketamine were related to achieving remission.

\section{MATERIALS AND METHODS}

\section{Participants}

The details of study cohorts and assessment procedures, and the GABA results were previously reported (Abdallah et al, 2015a; Abdallah et al, 2015b; Price et al, 2009). The NAc region of interest was not previously studied. All participants completed an informed consent process and an Institutional Review Board approved all study procedures. Cohort A included $26 \mathrm{HC}$ and $34 \mathrm{MDD}$ (Supplementary Table S1a) subjects with successful structural magnetic resonance imaging (MRI). Proton magnetic resonance spectroscopy $\left({ }^{1} \mathrm{H}\right.$ MRS) data were available on $20 \mathrm{HC}$ and $26 \mathrm{MDD}$ subjects. Study criteria for the Cohort A MDD group included a diagnosis of current MDD, no psychotropic treatment for at least 2 weeks, no substance or alcohol use disorders for at least 6 months, no current eating disorders, no unstable medical condition, and no history of mental retardation, or developmental, bipolar, or psychotic disorders. HC had no DSM-IV Axis I disorders. In cohort A, trait worry was assessed using Penn State Worry Questionnaire (PSWQ), and current depression and anxiety severity were assessed using Hamilton Depression Rating Scale (HDRS) and Hamilton Rating Scale for Anxiety (HAM-A), respectively. Cohort B included 16 MDD subjects (Supplementary Table S1b). Depression severity was assessed using Montgomery-Åsberg Depression Rating Scale (MADRS) as primary outcome, with remission defined as post-treatment MADRS $<10$ (Hawley et al, 2002). Participants completed MADRS and MRI scans at baseline (within $24 \mathrm{~h}$ prior to infusion) and $24 \mathrm{~h}$ following treatment with a single infusion of ketamine $0.5 \mathrm{mg} / \mathrm{kg}$ administered intravenously over $40 \mathrm{~min}$. Study criteria included current MDD diagnosis, treatment refractory to at least three antidepressants, no psychotropic treatment for at least 1 week (4 weeks for fluoxetine), no substance use disorder for at least 24 months, no unstable medical condition, and no history of bipolar or psychotic disorders. MRI contraindications were exclusionary for cohort A and B.

\section{Neuroimaging}

In cohort A, MRI and MRS acquisition were completed in one session on a 3.0T GE EXCITE magnet, with the following parameter: $\mathrm{MRI} \mathrm{T} 1$ sequence with $\mathrm{TR}=8.7 \mathrm{~ms}, \mathrm{TE}=1.8 \mathrm{~ms}$, flip angle $=7^{\circ}$, and voxel size $=0.9 \times 0.9 \times 1.5 \mathrm{~mm} ;{ }^{1} \mathrm{H}$ MRS $13 \mathrm{~min}$ GABA editing sequence from a single $\mathrm{mPFC}$ voxel $(2.5 \times 2.5 \times 3 \mathrm{~mm}$; see Supplementary Fig. S1 $)$ with 
$\mathrm{TR}=1500 \mathrm{~ms}, \mathrm{TE}=68 \mathrm{~ms}$, a 1024 sample points, $5-\mathrm{KHz}$ spectral width, and 256 interleaved excitations, which provided level of two metabolites: GABA and Glx (glutamate+glutamine). For additional details regarding MRS acquisition and processing see Abdallah et al (2015a); Price et al (2009). In cohort B, MRI scans were completed on a $3.0 \mathrm{~T}$ Siemens Trio system $(\mathrm{TR}=1200 \mathrm{~ms}, \mathrm{TE}=2.66 \mathrm{~ms}$, flip angle $=12^{\circ}$, voxel size $=1 \times 1 \times 1 \mathrm{~mm}$ ) within $24 \mathrm{~h}$ prior to treatment then repeated $24 \mathrm{~h}$ following ketamine infusion.

NAc volumes were estimated using the publically available Freesurfer image analysis suite (http://surfer.nmr.mgh.har vard.edu/). The fully automated recon-all pipeline, followed by routine quality checks, was completed. Briefly, this includes resampling to $1 \times 1 \times 1 \mathrm{~mm}$ images, intensity normalization, skull stripping, and segmentation of the gray/white matter and subcortical structures (including NAc). For cohort B, the longitudinal recon-all pipeline was used to enhance sensitivity in detecting changes overtime. This pipeline includes creation of an unbiased within subject template and resampling each time point scans to this base template to reduce variability (Reuter et al, 2012). For technical details regarding Freesurfer methods see Fischl (2012) and Fischl et al (2002). While blinded to the clinical status of participants, routine quality assurance checks and visual inspection were performed (Abdallah et al, 2013). In cohort B, three pre-treatment and two post-treatment scans were unsuccessful due to high motion artifacts. Freesurfer segmentation of the NAc has been previously shown to be successful in identifying neural correlates of the reward system (Wacker et al, 2009). Test-retest reliability using Freesurfer segmentation was previously reported at ICC $=$ 0.98 for NAC and ICC $=0.99$ for the hippocampus (Liem et al, 2015).

\section{Statistical Analyses}

The distribution of outcome measures was examined using test statistics and probability plots. Non-parametric tests and transformations were used as necessary. SEM is provided as an estimate of variance. Significance was set at $p \leqslant 0.05$. In cohort $\mathrm{A}$, we performed ANOVA, $t$-test and $\chi^{2}$ to assess age and gender differences between groups. As previously described (Abdallah et al, 2015a), the median split cutoff point of the mPFC GABA level was used to divide MDD subjects into two groups: GBD $(n=13$; ie, MDD with low GABA) and non-GBD ( $n=13$; ie, MDD with high GABA). Proportions of gray matter (GM), white matter (WM), and cerebrospinal fluid (CSF) for the mPFC voxel were computed (Supplementary Information). Percent GM, WM, and CSF did not differ between groups (all $p$-values $>0.05$ ). In addition, each of these variables were examined as covariates, but did no significantly alter the study findings.

General linear model (GLM) analyses were conducted to examine the diagnosis and subgroup effects on the NAc volume, controlling for intracranial volume (ICV) and age, followed by pairwise comparison with Bonferroni correction. These cross-sectional group comparisons were repeated without controlling for ICV or with using amygdala as covariate, which did not affect the study results (see Supplementary Information). Exploratory Spearman correlations in the MDD group, with FDR correction for multiple comparisons, examined the relationship between NAc and clinical severity, as well as the association between NAc and mPFC Glx levels.

To examine the effects of ketamine treatment on MADRS scores, and left and right NAc volumes, we constructed linear mixed models (LMM) with time (pre-treatment $v s$ posttreatment) as fixed factor, controlling for age as random factor. Follow-up exploratory analysis added remission status as a fixed factor to examine the effects of time, remission, and time ${ }^{\star}$ remission interactions, followed by pairwise comparison with Bonferroni correction. Comparable mixed models were constructed to examine the effect of hippocampal stratification. Results of the GLM and LMM analyses are provided as estimated marginal mean $\pm S E M$.

\section{RESULTS}

In cohort $\mathrm{A}$, there were no significant age and gender differences between MDD (41.7 \pm 2 years; 11 females) and HC groups (37.4 \pm 3 years; 14 females; all $p$-values $>0.05$ ). Similarly, age, gender, and tissue composition of the MRS voxel did not differ between subgroups (ie HC vs GBD vs non-GBD; all $p$ values $>0.1)$. MDD patients had moderate clinical severity with $\mathrm{HDRS}=20 \pm 1.1$, HAM-A $=22 \pm 1.5$, and $P S W Q=57 \pm 2.5$. In cohort $B$, average age was $45.9 \pm 2.7$ (7 females). Ketamine had a significant effect on depression severity (pre-treatment $\mathrm{MADRS}=34 \pm 1.2$; post-treatment MADRS $\left.=14 \pm 2.2 ; \mathrm{F}_{(1,14)}=62.6, p<0.001\right)$ and 6 of 16 (38\%) subjects met MADRS remission criteria, while 10 of $16(62 \%)$ showed more than $50 \%$ improvement.

\section{NAc Volume in MDD}

Controlling for age and intracranial volume, we found larger left NAc in the MDD $\left(599 \pm 18 \mathrm{~mm}^{3}\right)$ compared to HC $\left(489 \pm 21 \mathrm{~mm}^{3} ; \mathrm{F}_{(1,56)}=15.7, p=0.0002 ;\right.$ Cohen's $d=1.05$; Figure 1). The right NAc was not statistically different between MDD $\left(479 \pm 15 \mathrm{~mm}^{3}\right)$ and $\mathrm{HC}\left(440 \pm 17 \mathrm{~mm}^{3}\right.$; $\mathrm{F}_{(1,56)}=2.7, \quad p=0.10 ;$ Cohen's $d=0.44 ;$ Figure 1$)$. The interaction between diagnosis and hemisphere was statistically significant $\left(\mathrm{F}_{(1,56)}=6.2, p=0.02\right)$. Exploratory correlational analyses revealed a positive correlation between right NAc and PSWQ, which remained significant following correction for multiple comparisons $(r=0.48, d f=33$, corrected $p=0.02$; Supplementary Figure S2A), but no

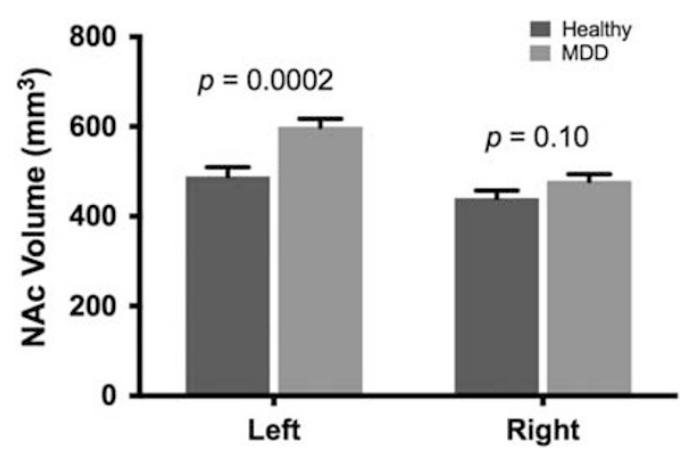

Figure I NAc volume in MDD vs HC. Larger left NAc in MDD subjects compared to $\mathrm{HC}$ (mean difference $\pm \mathrm{SEM}=|| \mid \pm 28 \mathrm{~mm}^{3}$ ). Right NAc was numerically larger in MDD (mean difference $\pm \mathrm{SEM}=39 \pm 23 \mathrm{~mm}^{3}$ ). $\mathrm{HC}$, healthy control; MDD, major depressive disorder; NAc, nucleus accumbens. 
correlations between left NAc and PSWQ or between left or right NAc and HDRS or HAM-A (all p-values $>0.05$ ).

In the follow-up subgroup analysis, we found a significant effect of group on the left $\left(\mathrm{F}_{(2,47)}=16.1, p=0.000005\right)$ and right $\operatorname{NAc}\left(\mathrm{F}_{(2,47)}=5.7, p=0.006\right)$, with post hoc pairwise comparisons showing larger left and right NAc in the nonGBD compared to GBD (left: Cohen's $d=1.48$; right: Cohen's $d=0.95$ ) and HC (left: Cohen's $d=1.90$; right: Cohen's $d=1.12$ ), but no significant differences between GBD and HC (Figure 2). The interaction between subgroup status and hemisphere was statistically significant $\left(\mathrm{F}_{(2,57)}=6.2, p=0.05\right)$. Examining the relationship between NAc and Glx showed positive correlation between mPFC Glx and right NAc $(r=0.44, d f=25$, corrected $p=0.05$; Supplementary Fig. S2B), but not left NAc $(r=0.15$, corrected $p=0.47)$. To examine whether mPFC Glx mediated the link between NAc hypertrophy and severity of worry symptoms, we conducted a mediation analysis with bootstrapping $(n=10000)$. We found that mPFC Glx did not mediate the relationship between NAc volume and PSWQ $(\mathrm{p}=0.52)$.

\section{NAc Volume \& Ketamine Treatment}

The LMM revealed a small, but statistically significant, effect of time on the left $\left(\mathrm{F}_{(1,11)}=5.4, p=0.04\right)$, but not on the right NAc $(p=0.52)$, showing reduction in left NAc volume following treatment (Figure 3a; Supplementary Table S2). We found no significant hemisphere by time interaction $(p=0.2)$. Follow-up exploratory analysis, examining whether the left NAc changes were affected by treatment response, found significant time effect $\left(F_{(1,11)}=7.2, p=0.02\right)$ and a trend for time ${ }^{\star}$ remission interaction $\left(\mathrm{F}_{(1,11)}=5.4, p=0.098\right)$, but no remission effects $\left(\mathrm{F}_{(1,15)}=2.2, p=0.16\right)$, with significant reduction in left NAc in remitters $(p=0.01)$, but not non-remitters $(p=0.53$; Figure $3 b)$.

Considering preclinical evidence relating the rapid antidepressant effects of ketamine to its reversal of depressionrelated gray matter deficits in the hippocampus, we conducted an exploratory analysis examining the effects of ketamine on left and right hippocampal volumes. The LMM in the left hippocampus revealed a significant time ${ }^{\star}$ remission interaction $\left(\mathrm{F}_{(1,12)}=5.3, p=0.04\right)$, with a trend of increased volume in the remitters (Figure 4 ). We found no significant time $\left(\mathrm{F}_{(1,12)}=0.7, p=0.44\right)$ or remission effects $\left(\mathrm{F}_{(1,16)}=0.1\right.$, $p=0.80)$. There were no significant effects of time $\left(\mathrm{F}_{(1,11)}=0.05, p=0.83\right)$, remission $\left(\mathrm{F}_{(1,16)}=0.01, p=0.94\right)$, or time* remission interaction $\left(\mathrm{F}_{(1,11)}=0.8, p=0.40\right)$ in the right hippocampus. There was no significant hemisphere by time interaction $(p=0.8)$. See online Supplementary Information for exploratory analyses related to anhedonia.

\section{Stratification Based on Hippocampal Volume}

The lack of GABA measure in Cohort B hindered our ability to test whether the ketamine effects on the NAc were more profound in a subgroup of non-GBD patients. Therefore, we conducted an exploratory analysis in which MDD patients were stratified based on the median split of baseline total hippocampal volume (ie, 17 vs 17 in Cohort $\mathrm{A}$ and 8 vs 8 in Cohort B). For completeness, we have also included the stratification based on the left hippocampal volume,

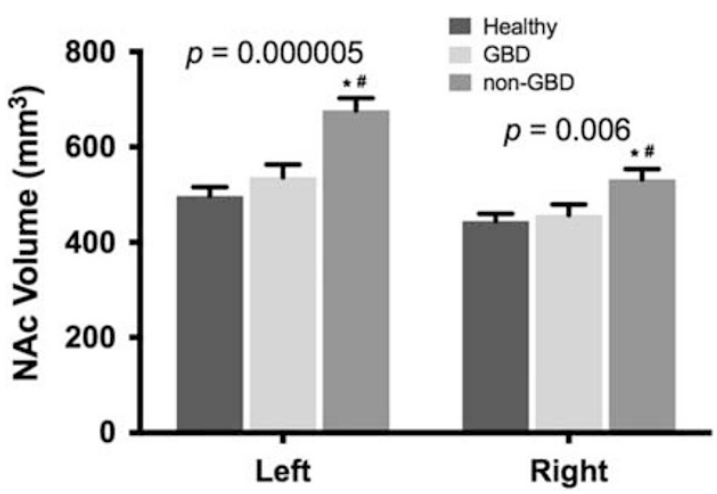

Figure 2 NAc volume in GBD vs non-GBD vs HC. Significant group effect with post hoc analyses showing larger left and right NAc in non-GBD compared to GBD and HC. * $p \leqslant 0.05$ compared to $\mathrm{HC}$. ${ }^{*} p \leqslant 0.05$ compared to GBD. GBD=glutamate-based depression; $\mathrm{HC}$, healthy control; NAc, nucleus accumbens.

considering that depression and ketamine effects were most evident in the left hemisphere.

In Cohort $\mathrm{A}$, we found a significant subgroup effect on left, but not right, NAc volumes using either total or left hippocampus for stratification (for details see Supplementary Fig. S3). In Cohort B, ketamine reduced left and right NAC volumes in the subgroup with high left hippocampal volumes, but no significant changes were found in the subgroup with low total or left hippocampal volumes (for details see Figure 5).

\section{DISCUSSION}

Consistent with preclinical findings of depression-related hypertrophy in the NAc (Campioni et al, 2009; Christoffel et al, 2011; Christoffel et al, 2012; Muhammad et al, 2012; Warren et al, 2014), we found increased NAc volumes in the MDD group compared to HC. These NAc volume abnormalities were significant only in the left hemisphere, with a trend for larger right NAc in MDD. The data revealed that the NAc alterations in MDD are primarily limited to the subgroup of MDD patients with no glutamate-based abnormalities. Similarly, mPFC Glx positively correlated with right NAc volume. Together, these data suggest that the preclinical evidence of stress-induced synchronous NAc hypertrophy and hippocampal hypotrophy is not paralleled by synchronous gray matter abnormalities in both structures in depressed humans. In MDD, the study evidence supports the division of patients into subgroups with amino acid-based (ie, GBD) vs monoamine-based (ie, non-GBD) gray matter abnormalities (for additional discussion see Supplementary Information).

Within MDD patients, there were no significant correlations between NAc volumes and current severity of depression or anxiety. However, individuals with high trait worry scores exhibited relatively larger right NAc volumes. One possible interpretation of the latter finding is that trait worry may be related to failure in error detection and correction, a function that was recently attributed to NAc in humans (Munte et al, 2007). An alternative interpretation could be the hypothesized causal role between worry and anhedonia (Burrows-Kerr, 2015). Comparable to preclinical 

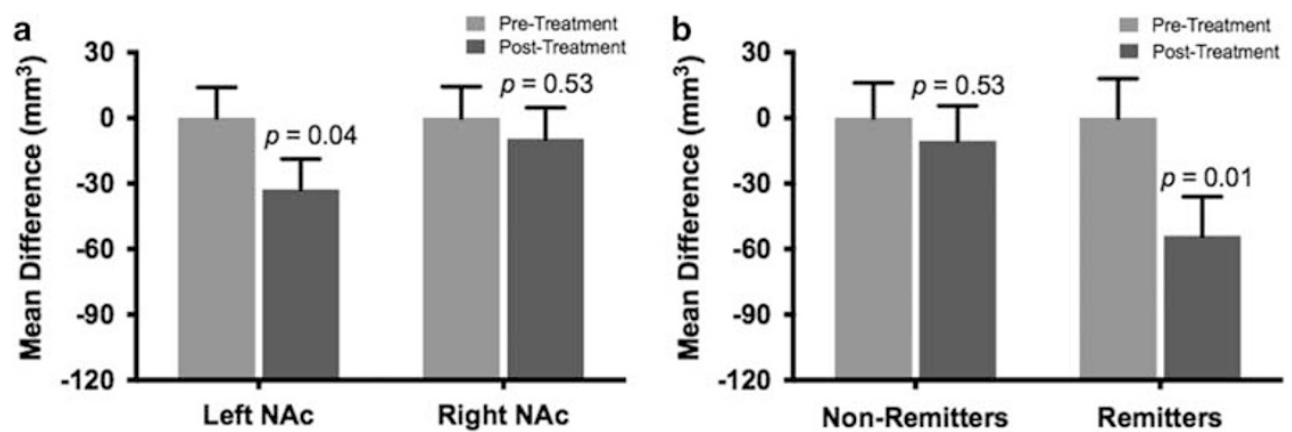

Figure 3 Ketamine effects on the nucleus accumbens (NAc). In the treatment cohort, a linear mixed model revealed a modest but statistically significant reduction in left NAc volume post ketamine treatment (mean difference $\pm \mathrm{SEM}=33 \pm 14 \mathrm{~mm}^{3}$ ). Ketamine had no significant effect on the right $\mathrm{NAc}$ volume (a). Further exploring the left NAc changes by adding the remission status to the model revealed a treatment-by-remission interaction trend, such that ketamine reduced left NAc in remitters (mean difference $\pm S E M=54 \pm 18 \mathrm{~mm}^{3}$ ), but not in non-remitters (b).

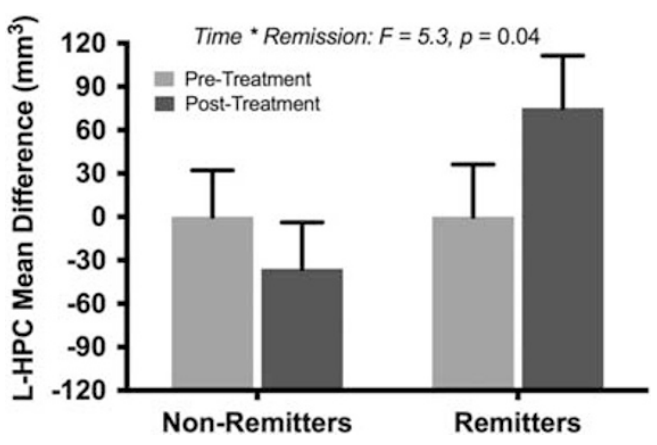

Figure 4 Ketamine effects on the hippocampus (HPC). In the treatment cohort, a linear mixed model revealed a treatment-by-remission interaction, such that ketamine increased left HPC in remitters.

data showing ketamine-induced normalization of NAc hypertrophy and hippocampal hypotrophy (Melo et al, 2015), we found rapid reduction in left NAc, but increase hippocampal, volumes following ketamine treatment, particularly in patients who achieve remission. In line with the GBD model, subgrouping patients based on hippocampal volumes was statistically significant showing a pattern in which the higher NAc volumes were in the subgroup with large hippocampus. However, the GABA stratification effects appeared to be more profound. Interestingly, the preliminary analysis examining the effects of hippocampal stratification suggested that the ketamine-induced reduction in the NAc is mostly restricted to the subgroup with high hippocampal volume. However, the ketamine-related plasticity findings and hippocampal stratification should be interpreted with extreme caution considering the exploratory nature, the relatively small sample and the need for replication.

While underscoring the preliminary nature of the ketamine findings, the results add to extensive preclinical evidence, and nascent human studies, directly relating the rapid acting antidepressant effect of ketamine to region specific synaptogenic alterations. Although the longitudinal findings are anticipated by extent preclinical evidence (Melo et al, 2015; Reus et al, 2013), it is still remarkable-and perhaps dubious-that the MRI estimates of NAc and hippocampal volumes were sufficiently sensitive to detect these rapid changes following treatment. Though we reiterate that the reader should be cautious not to over interpret these results, we here describe related evidence that support these intriguing findings. First, there is the intuitive concept that structural brain changes would require prolonged time to occur, for example aging- and stress-induced neuronal atrophy would require weeks to years (McEwen et al, 2015). However, accumulating evidence suggest that neuronal plasticity is an often prompt continuous process that could be functionally and micro-structurally evident within minutes to hours, for example neuronal atrophy induced by a 21-day of chronic unpredictable stress was fully reversed within $24 \mathrm{~h}$ of ketamine infusion in rodents (Li et al, 2011). Second, there is the question whether these 21-day stressrelated microstructural changes would be evident with gross MRI estimates of volume. Yet, this was already demonstrated in rodents where the MRI estimates of gray matter volumes were able to capture the percent changes in microstructural neuronal atrophy following a 21-stress paradigm (Kassem et al, 2013). Third, these preclinical findings may not necessarily translate into humans, raising the question whether MRI estimates could be sensitive to detect within hours structural changes. In addition to the current data demonstrating rapid volumetric changes that were specifically related to treatment efficacy, a recent study provides another example of rapid gray matter volumetric changes demonstrated by MRI (Tost et al, 2010). The dopamine antagonist haloperidol was found to reduce striatal volume, as estimated by MRI, within $2 \mathrm{~h}$ of intravenous administration. These volumetric changes were associated with the drug-induced neuroleptic extrapyramidal effects and were believed to reflect preclinical evidence of microstructural changes (Tost et al, 2010).

Considering that the stress-induced NAc hypertrophy is related to perturbation in dopaminergic neurons, a putative mechanism for the ketamine-induced NAc reduction could be a direct modulation of dopaminergic neurons by ketamine. Ketamine infusion in rodents increase spontaneous activation of dopaminergic neurons in the VTA and increase extracellular dopamine in the NAc (Witkin et al, 2016). In addition, dopamine receptors antagonists block the antidepressant-like effects of ketamine in rodents (Belujon and Grace, 2014; Li et al, 2015). At the network level, ketamine has been shown in rodents to restore the stressinduced dopaminergic perturbation and synaptic dysfunction (Belujon and Grace, 2014). These changes partly involve 

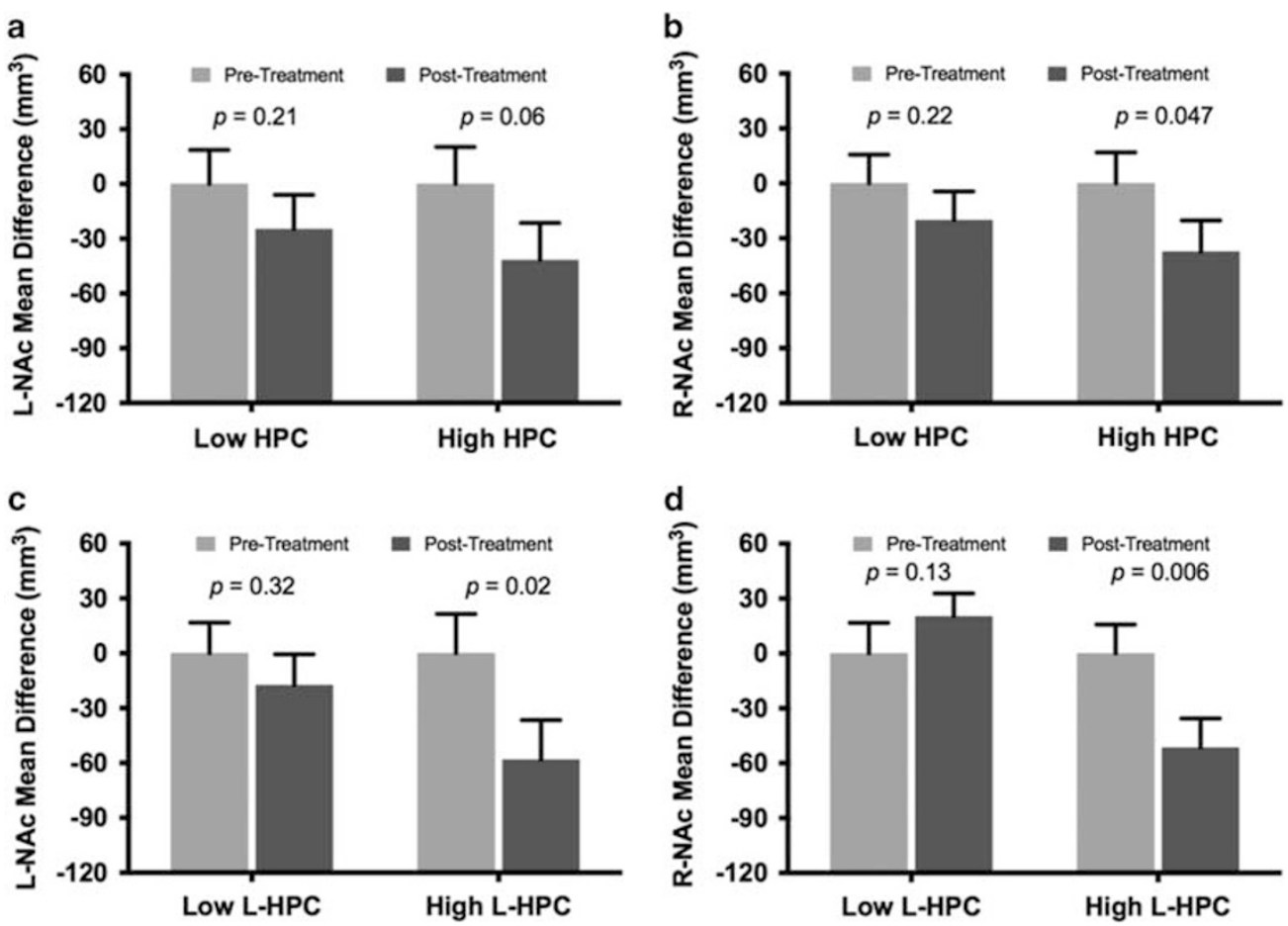

Figure 5 Ketamine effects on the nucleus accumbens (NAc) volume in major depressive disorder (MDD) subgroups based on hippocampal (HPC) volume. In panel ( $\mathrm{a}$ and $\mathrm{b}$ ), patients were stratified based on total HPC volume using median split. This analysis showed significant reduction of right NAc (R-NAc), but not left (L-NAc), in the high HPC subgroup. The were no significant L-NAc or R-NAc changes in the low HPC subgroup. In (c and d), patients were stratified based on left HPC (L-HPC) volume using median split. This analysis showed significant reduction in L-NAc and right NAc (R-NAc) volumes in the high L-HPC subgroup. The were no significant L-NAc or R-NAc changes in the low L-HPC subgroup.

the hippocampal input to the NAc and could be blocked by D1 receptor antagonists (Belujon and Grace, 2014).

Limitations of the current study include the secondary nature of the approach. The parent studies, from which the biological and behavioral measures were extracted, were not designed to interrogate the NAc abnormalities in MDD. For example, it would have been more optimal if both the cross sectional (MDD vs $\mathrm{HC}$ ) and longitudinal study aims (effects of ketamine) were investigated in the same cohort (ie, treatment of the MDD and repeated scans in both MDD and HC). Future studies would also greatly benefit from using a Research Domain Criteria (RDoC) approach to investigate the reward system, while capitalizing on the unique ketamine paradigm of rapid behavioral response, large effect size, and extensive literature of the drug mechanisms which would facilitate the integration of new findings in previously developed working models. Furthermore, while the multimodal MRI/MRS approach is a strength of the cohort A study, the MRS scans were not conducted in cohort B. Moreover, the addition of functional and diffusion MRI in future studies might further unravel the role of NAc in the neurobiology and treatment of MDD. The use of median split is consistent with our previous reports, however, this limit the generalizability of the findings. While cortical GABA in MDD has been relatively consistent finding, a major challenge in the field is to identify a cutoff value that could be used to stratify patients. Another limitation is that the correlation between NAc volume and Glx may not necessarily reflect glutamate neurotransmission as Glx is a total level of glutamate and glutamine, both of which are primarily intracellular rather than intrasynaptic (Abdallah et al, 2014). The sample size of cohort B could be considered a limitation. Among the current study strengths are the use of: well-characterized psychotropic-free cohorts; crosssectional and longitudinal approaches; well validated structural and neuroimaging methods; and the capitalization on the ketamine paradigm, which exerts rapid behavioral and biological effects within $24 \mathrm{~h}$ of treatment.

\section{CONCLUSIONS}

This study provides the first in vivo in-human evidence of enlarged NAc volume in MDD. We also demonstrated that the NAc volumetric abnormalities are absent in patients with prominent glutamate based abnormalities. Together the results supported the notion that patients with and without NAc abnormalities may have differing underlying pathology that could be targeted with differential antidepressant treatment, offering hope for individualized medicine, as well as potentially shedding light on putative causes of the pervasive failure of numerous antidepressant trials. Finally, the longitudinal data revealed a pattern of rapid normalization of NAc and hippocampal volumes limited to patients who achieved remission, an intriguing finding that awaits replication prior to any firm conclusions.

\section{FUNDING AND DISCLOSURE}

CGA has served as a consultant or on advisory boards for Genentech and Janssen. He also serves as editor for the 
journal Chronic Stress published by SAGE Publications. JDC received grant support from NIMH, NYSTEM, GlaxoSmithKline, Pfizer, and Alexza Pharmaceuticals. He is on the Pfizer advisory board and gives talks for BMS, AstraZeneca, GSK, and Pfizer. SJM: In the past 12 months, Dr Mathew has received consulting fees from Acadia, Cerecor, Otsuka, and Valeant, and serves on an Advisory Board for VistaGen Therapeutics. He has received research support from Janssen Research \& Development. The remaining authors declare no conflict of interest.

\section{ACKNOWLEDGMENTS}

We thank the subjects who participated in this study for their invaluable contribution. This work was supported by the Clinical Neuroscience Division National Center for PTSD, K23 MH-101498, Brain and Behavior Foundation (NARSAD New Investigator Award; CGA), R01 MH07895 (DCS), K23 MH-069656, MO1 RR-00071, R01 MH-081870. The Johnson Family Chair for Research, and by the use of resources and facilities at the Michael E. DeBakey VA Medical Center, Houston, Texas (SJM). The content is solely the responsibility of the authors and does not necessarily represent the official views of the National Institutes of Health or the Department of Veterans Affairs.

\section{REFERENCES}

Abdallah CG, Adams TG, Kelmendi B, Esterlis I, Sanacora G, Krystal JH (2016a). Ketamine's mechanism of action: a path to rapid-acting antidepressants. Depress Anxiety 33: 689-697.

Abdallah CG, Averill LA, Collins KA, Geha P, Schwartz J, Averill C et al (2016b). Ketamine treatment and global brain connectivity in major depression. Neuropsychopharmacology (doi:10.1038/npp.2016.186).

Abdallah CG, Coplan JD, Jackowski A, Sato JR, Mao X, Shungu DC et al (2013). A pilot study of hippocampal volume and $\mathrm{N}$-acetylaspartate (NAA) as response biomarkers in riluzoletreated patients with GAD. Eur Neuropsychopharmacol 23: 276-284.

Abdallah CG, Jackowski A, Sato JR, Mao X, Kang G, Cheema R et al (2015a). Prefrontal cortical GABA abnormalities are associated with reduced hippocampal volume in major depressive disorder. Eur Neuropsychopharmacol 25: 1082-1090.

Abdallah CG, Jiang L, De Feyter HM, Fasula M, Krystal JH, Rothman DL et al (2014). Glutamate Metabolism in Major Depressive Disorder. Am J Psychiatry 171: 1320-1327.

Abdallah CG, Salas R, Jackowski A, Baldwin P, Sato JR, Mathew SJ (2015b). Hippocampal volume and the rapid antidepressant effect of ketamine. J Psychopharmacol 29: 591-595.

Abdallah CG, Sanacora G, Duman RS, Krystal JH (2015c). Ketamine and rapid-acting antidepressants: a window into a new neurobiology for mood disorder therapeutics. Annu Rev Med 66: 509-523.

Averill LA, Purohit P, Averill CL, Boesl MA, Krystal JH, Abdallah CG (2016). Glutamate dysregulation and glutamatergic therapeutics for PTSD: evidence from human studies. Neurosci Lett (doi:10.1016/j.neulet.2016.11.064).

Belujon P, Grace AA (2014). Restoring mood balance in depression: ketamine reverses deficit in dopamine-dependent synaptic plasticity. Biol Psychiatry 76: 927-936.

Burrows-Kerr R. Repetitive negative thought and anhedonia: a systematic review (literature review); Repetitive negative thought and reward sensitivity (empirical paper). University of Exeter: Exeter Devon, UK, 2015.
Campioni MR, Xu M, McGehee DS (2009). Stress-induced changes in nucleus accumbens glutamate synaptic plasticity. J Neurophysiol 101: 3192-3198.

Chaudhury D, Walsh JJ, Friedman AK, Juarez B, Ku SM, Koo JW et al (2013). Rapid regulation of depression-related behaviours by control of midbrain dopamine neurons. Nature 493: 532-536.

Christoffel DJ, Golden SA, Dumitriu D, Robison AJ, Janssen WG, $\mathrm{Ahn} \mathrm{HF}$ et al (2011). IkappaB kinase regulates social defeat stressinduced synaptic and behavioral plasticity. $J$ Neurosci 31: 314-321.

Christoffel DJ, Golden SA, Heshmati M, Graham A, Birnbaum S, Neve RL et al (2012). Effects of inhibitor of kappaB kinase activity in the nucleus accumbens on emotional behavior. Neuropsychopharmacology 37: 2615-2623.

Coplan JD, Gopinath S, Abdallah CG, Berry BR (2014). A neurobiological hypothesis of treatment-resistant depression mechanisms for selective serotonin reuptake inhibitor nonefficacy. Front Behav Neurosci 8: 189.

Duman RS, Aghajanian GK, Sanacora G, Krystal JH (2016). Synaptic plasticity and depression: new insights from stress and rapid-acting antidepressants. Nat Med 22: 238-249.

Fischl B (2012). FreeSurfer. Neuroimage 62: 774-781.

Fischl B, Salat DH, Busa E, Albert M, Dieterich M, Haselgrove C et al (2002). Whole brain segmentation: automated labeling of neuroanatomical structures in the human brain. Neuron 33: 341-355.

Hawley CJ, Gale TM, Sivakumaran T, Hertfordshire Neuroscience Research group (2002). Defining remission by cut off score on the MADRS: selecting the optimal value. J Affect Disord 72: 177-184.

Kassem MS, Lagopoulos J, Stait-Gardner T, Price WS, Chohan TW, Arnold JC et al (2013). Stress-induced grey matter loss determined by MRI is primarily due to loss of dendrites and their synapses. Mol Neurobiol 47: 645-661.

Kempton MJ, Salvador Z, Munafo MR, Geddes JR, Simmons A, Frangou $S$ et al (2011). Structural neuroimaging studies in major depressive disorder. Meta-analysis and comparison with bipolar disorder. Arch Gen Psychiatry 68: 675-690.

Krishnan V, Han MH, Graham DL, Berton O, Renthal W, Russo SJ et al (2007). Molecular adaptations underlying susceptibility and resistance to social defeat in brain reward regions. Cell 131: 391-404.

Li N, Liu RJ, Dwyer JM, Banasr M, Lee B, Son H et al (2011). Glutamate N-methyl-D-aspartate receptor antagonists rapidly reverse behavioral and synaptic deficits caused by chronic stress exposure. Biol Psychiatry 69: 754-761.

Li Y, Zhu ZR, Ou BC, Wang YQ, Tan ZB, Deng CM et al (2015). Dopamine D2/D3 but not dopamine D1 receptors are involved in the rapid antidepressant-like effects of ketamine in the forced swim test. Behav Brain Res 279: 100-105.

Liem F, Merillat S, Bezzola L, Hirsiger S, Philipp M, Madhyastha T et al (2015). Reliability and statistical power analysis of cortical and subcortical FreeSurfer metrics in a large sample of healthy elderly. Neuroimage 108: 95-109.

McEwen BS, Bowles NP, Gray JD, Hill MN, Hunter RG, Karatsoreos IN et al (2015). Mechanisms of stress in the brain. Nat Neurosci 18: 1353-1363.

Melo A, Kokras N, Dalla C, Ferreira C, Ventura-Silva AP, Sousa N et al (2015). The positive effect on ketamine as a priming adjuvant in antidepressant treatment. Transl Psychiatry 5: e573.

Muhammad A, Carroll C, Kolb B (2012). Stress during development alters dendritic morphology in the nucleus accumbens and prefrontal cortex. Neuroscience 216: 103-109.

Munte TF, Heldmann M, Hinrichs H, Marco-Pallares J, Kramer UM, Sturm V et al (2007). Nucleus Accumbens is Involved in Human Action Monitoring: Evidence from Invasive Electrophysiological Recordings. Front Hum Neurosci 1: 11. 
Nestler EJ (2015). Role of the Brain's Reward Circuitry in Depression: Transcriptional Mechanisms. Int Rev Neurobiol 124: 151-170.

Phillips ML, Chase HW, Sheline YI, Etkin A, Almeida JR, Deckersbach $\mathrm{T}$ et al (2015). Identifying predictors, moderators, and mediators of antidepressant response in major depressive disorder: neuroimaging approaches. Am J Psychiatry 172: 124-138.

Price RB, Shungu DC, Mao X, Nestadt P, Kelly C, Collins KA et al (2009). Amino acid neurotransmitters assessed by proton magnetic resonance spectroscopy: relationship to treatment resistance in major depressive disorder. Biol Psychiatry 65: 792-800.

Reus GZ, Abelaira HM, dos Santos MA, Carlessi AS, Tomaz DB, Neotti MV et al (2013). Ketamine and imipramine in the nucleus accumbens regulate histone deacetylation induced by maternal deprivation and are critical for associated behaviors. Behav Brain Res 256: 451-456.

Reuter M, Schmansky NJ, Rosas HD, Fischl B (2012). Withinsubject template estimation for unbiased longitudinal image analysis. Neuroimage 61: 1402-1418.

Russo SJ, Nestler EJ (2013). The brain reward circuitry in mood disorders. Nat Rev Neurosci 14: 609-625.
Tost H, Braus DF, Hakimi S, Ruf M, Vollmert C, Hohn F et al (2010). Acute D2 receptor blockade induces rapid, reversible remodeling in human cortical-striatal circuits. Nat Neurosci 13: 920-922.

Wacker J, Dillon DG, Pizzagalli DA (2009). The role of the nucleus accumbens and rostral anterior cingulate cortex in anhedonia: integration of resting EEG, fMRI, and volumetric techniques. Neuroimage 46: 327-337.

Walsh JJ, Friedman AK, Sun H, Heller EA, Ku SM, Juarez B et al (2014). Stress and CRF gate neural activation of BDNF in the mesolimbic reward pathway. Nat Neurosci 17: 27-29.

Warren BL, Sial OK, Alcantara LF, Greenwood MA, Brewer JS, Rozofsky JP et al (2014). Altered gene expression and spine density in nucleus accumbens of adolescent and adult male mice exposed to emotional and physical stress. Dev Neurosci 36: 250-260.

Witkin JM, Monn JA, Schoepp DD, Li X, Overshiner C, Mitchell SN et al (2016). The rapidly acting antidepressant ketamine and the mGlu2/3 receptor antagonist LY341495 rapidly engage dopaminergic mood circuits. J Pharmacol Exp Ther 358: 71-82.

Wook Koo J, Labonte B, Engmann O, Calipari ES, Juarez B, Lorsch $\mathrm{Z}$ et al (2016). Essential role of mesolimbic brain-derived neurotrophic factor in chronic social stress-induced depressive behaviors. Biol Psychiatry 80: 469-478.

Supplementary Information accompanies the paper on the Neuropsychopharmacology website (http://www.nature.com/npp) 